Original Article

\title{
SYNTHESIS AND ANTICANCER SCREENING OF TRIAZINE ANALOGUES
}

\section{ANSHULY TIWARI, SIDDHARTH J. MODI, KAKASAHEB R. MAHADIK, MUGDHA R. SURYAWANSHI*}

Department of Pharmaceutical Chemistry, Poona College of Pharmacy, Bharati Vidyapeeth (Deemed to be University), Pune, Maharashtra, India Email: mugdha.rs@gmail.com

Received: 03 Jul 2018 Revised and Accepted: 06 Mar 2019

ABSTRACT

Objective: The study was aimed to investigate the cytotoxic effect of $S$-5H-[1,2,4]-triazino $(5,6-b)$ indol-3-yl-3,4-phenylethane-thioate derivatives as epidermal growth factor Receptor (EGFR) inhibitors.

Methods: In the present study 14 novel triazine analogues were synthesized and characterized using different spectroscopic techniques such as FTIR, NMR and Mass Spectroscopy. The anticancer activity was performed using MCF-7 (breast cancer) and K-562 (leukaemia) cell lines. Further, molecular docking was carried out using Vlife Molecular Docking Software (MDS) on crystal structure of epidermal growth factor receptor (EGFR) to identify the binding mode of interaction with an active site.

Results: Compounds MA-7, MA-8, MA-12, MA-13 and MA-14 show potent activity against cancer cell lines in the range of $<10$ to $84.4 \mu \mathrm{g} / \mathrm{ml}$. Further molecular docking on EGFR also supports that there is a strong correlation between in silico and in vitro biological activity. The results of this study may be further useful for lead optimization process.

Conclusion: The results of this study indicates that the synthesized triazine analogues can give a potential lead as an anticancer agent.

Keywords: Cancer, Triazine Analogues, MCF-7, K-562, Anticancer activity, EGFR

(C) 2019 The Authors. Published by Innovare Academic Sciences Pvt Ltd. This is an open-access article under the CC BY license (http://creativecommons.org/licenses/by/4.0/) DOI: http://dx.doi.org/10.22159/ijpps.2019v11i4.28275

\section{INTRODUCTION}

Cancer is a condition that refers to a state in which the cell present in the body gone awry and reproduced in an uncontrollable manner [1]. The healthy tissues and organs that are present in the body are then proliferated or intrude get destroyed [2]. The growth of the cancer begins with one part/specific organ of the body and further spread into the entire body. Cancerous cells segregate and mimic themselves and could be present in the form of a cluster of infected cells that are known as malignancy (tumor) [3]. The symptom of cancer originated from the coercing, pulverizing and destroying the surrounding healthy cells and tissues [4]. The immune system has crucial role in the management and elimination of early cancer or premalignant cells because the immune system is associated with increased incidences of a variety of cancer [5]. In women, breast cancer is a more common while, colorectal, prostate and lung cancer occurs most commonly in men [6]. Development of breast cancer is seen most commonly in the cells present in the inner linings of the milk ducts and the lobules, which are the major source for the supply of the milk in the ducts [7]. Factors emerging risk of breast cancer are obesity, decrease in physical exertion, alcohol intake, hormone replacement therapy at the ongoing menopause, early age menstruation, late pregnancy or no pregnancy at all. Approximately $5 \%$ of cases are occurring due to the inherited gene expression from the patient's parents. Risk factors for cancer also involve breast cancer genes namely BRCA-1 and BRCA-2 among others [8]. The other type of cancer we have focused is leukaemia which is known as blood cancer and starts with the bone marrow and results into the multiplication of white blood cells (WBCs) [9]. With the progression of leukaemia, the infected cells crowed out to suppress the growth of normal cells. Sometimes some hairy thin projections are seen in an infrequent chronic leukaemia which is called as hairy cell leukaemia (HCL) that starts with the bone marrow and the accumulation of these hairy cells present in the bone marrow, liver and spleen. Though hairy cell leukaemia strikes the WBC, the enlargement in the lymph nodes doesn't observed [10]. Although there are no clear symptoms for the development of HCL in the early stage.

EGFR receptor is devided in to four receptors namely EGFR (ErbBI/HER-1\{Human Epidermal Growth Factor Receptor-1\}) ErbB-II
(Neu, HER-2), ErbB-III (HER-3) and ErbB-IV (HER-4) [11]. RTK family contains an extracellular ligand-binding domain that is a hydrophobic transmembrane domain, and cytoplasmic tyrosine kinase domain [12]. A knockout model for EGFR shows the lethality of embryo and defects in tissues or organs. The abnormal activation of EGFR is expressed in many types of specific diseases [13]. The pleiotropic function of EGFR signaling helps to develop and regulate various physiological functions of the body [14]. The incrimination of various types of disorders has been defined by the exponential expression of EGFR. There are several reports are available in the literature suggesting that aromatic and heteroaromatic substitution over chosen triazine ring moiety shows potent anticancer activity, therefore, here in this study the substituted benzoic acids were used and were studied as anticancer agents [15]. The main objective of the study is to perform synthesis and anticancer screening of synthesized compounds using various cell lines.

\section{MATERIALS AND METHODS}

\section{Chemicals and reagents}

Raw materials were purchased from Loba Chemie, Sigma Aldrich, Merck (India), Sisco Research Laboratory SRL (India) and Spectrochem Pvt. Ltd. and were of the synthetic grade. Melting point detection was conducted by using Veego VMP-D digital apparatus. Silica gel precoated F-254 TLC plates were used for monitoring the progress of reaction; further detection of the retention factor is obtained by either staining by iodine or visualizing under UV-light chamber. Fourier transform infrared (FT-IR) spectra were obtained by making sample cuvettes in anhydrous potassium bromide (KBr) on "JASCO FTIR 4100" and are reported in $\mathrm{cm}^{-1}$. ${ }^{1} \mathrm{H}-\mathrm{NMR}$ spectra were illustrated on "Bruker Avance having a frequency of $400 \mathrm{MHz}$ using DMSO-D 6 in the presence of an internal standard tetramethylsilane (TMS).

Step I: General procedure of synthesis of 1, 2, 4-triazino-(5, 6b)-indole-3-thione

To a mixture of Isatin $(0.01 \mathrm{~mol})$ and thiosemicarbazide $(0.01 \mathrm{~mol})$, distilled water $(300 \mathrm{ml})$ containing potassium carbonate $(0.04 \mathrm{~mol})$ was taken in a round bottom flask. The reaction flask was refluxed 
for $4 \mathrm{~h}$ during which an initial intense red coloured solution turned orange. Upon cooling at room temperature, the reaction mixture was filtered and the residual filtrate obtained was acidified with glacial acetic acid to precipitate the yellow coloured product. Precipitates obtained by filtration were 3 to 4 times with water to obtain the crude product. The crude product was then stirred with methanol (25 $\mathrm{ml}$ ) for $2 \mathrm{~h}$, filtered, dried and subsequently re-crystallized to furnish the final product $(1.34 \mathrm{~g}, 66.3 \%)$ [16].

\section{Scheme of synthesis}

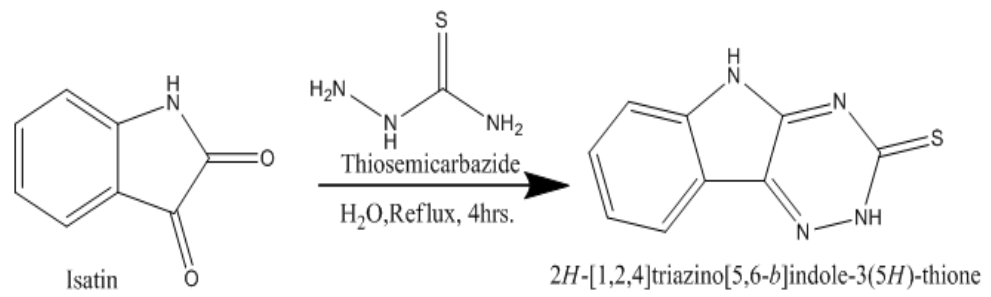

(1)

Step I

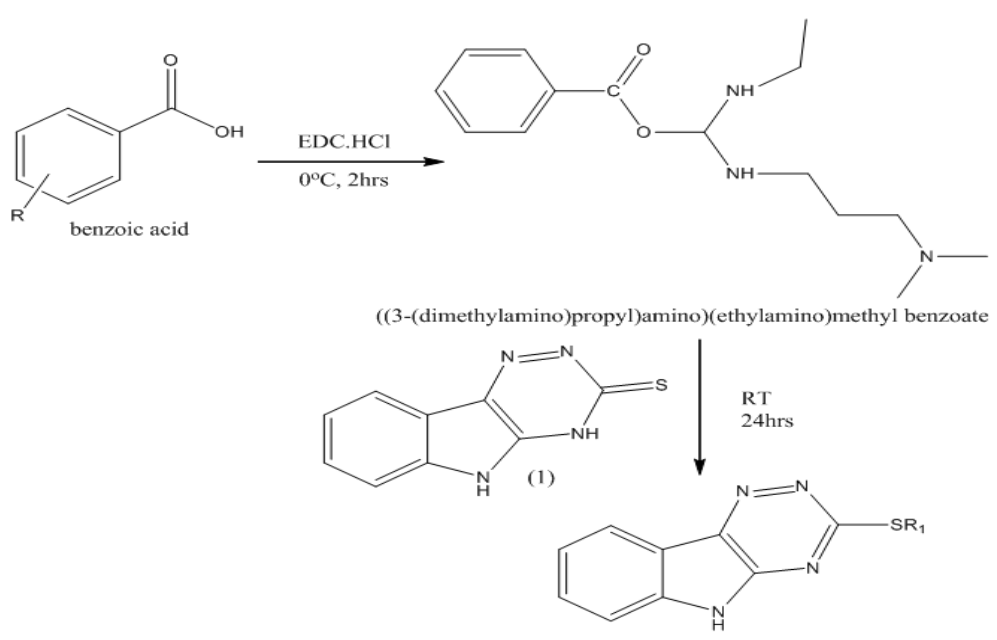

Substituted $[1,2,4]$ triazino[ $[5,6-b]$ indole-3-thione

(2)

\section{Step II}

Step II General procedure of synthesis of $S-5 H-[1,2,4]$-triazino(5,6-b)indol-3-yl-3,4-biphenylethanethioate (MA-1 to MA-14)

Substituted benzoic acid (0.01 mol) and 1-Ethyl-3-(3-dimethylaminopropyl) carbodiimide (EDC. $\mathrm{HCl}$ ) was taken in $100 \mathrm{ml}$ round bottom flask containing $N-N$ dimethylformamide (DMF) as a solvent and the reaction mixture was then agitated for $2 \mathrm{~h}$ at $0{ }^{\circ} \mathrm{C}$. Later, on the completion of $2 \mathrm{~h}$ the crude product (reaction step I (1)) was added (0.01 mol). The reaction mixture was then agitated at room temperature for $24 \mathrm{~h}$. The reaction mixture was extracted with ethyl acetate (EtOAc), was filtered, dried and recrystallized with chloroform.

\section{Molecular docking studies}

\section{Ligand preparation}

4-biphenylethanethioate derivatives were drawn as the template for building the compounds present in the dataset of V-Life molecular docking software (MDS) version 3.5. The geometry of the ligands was modified by the minimization of energy with the use of Merck Molecular force field (MMFF94) and charges for atoms was applied until a $0.001 \mathrm{kcal} / \mathrm{mol} / \AA$ gradient was reached, perpetuating the rigid structure template throughout the process of minimization of energy [17].

\section{Grid generation}

Using the Vlife molecular docking software (MDS) version 3.5 the grid map was centralized at the active site of the protein.
The grid map was streamlined at the residues namely ASP 212 (A), LYS 179 (A), HIS 180(A), ARG 174(A), ASP 269(A), HIS 268 (A), TYR59(A), GLN 63 (A), ASP 273 (A) respectively which were then forecasted from the ligpot.

\section{Docking file generation}

On the activation of the receptor EGFR (1M17) signalling pathways are strongly activated for anticancer activity. Hence, EGFR-1M17 was selected as a biological target for the docking study. The crystallized structure was retrieved from the Protein Data Bank (PDB) \{www. rcsb. org/pdb\}. The receptor was optimized; saved as. mol file and used for docking simulation. Two dimensional structures of the synthesized compounds were drawn and then transfigured into the 3D structures with Vlife MDS. Energy minimization of the 3D structures was performed up to the rms gradient of value 0.01 by the use of MMFF94. For all the conformers, energy minimization method was performed. The selection of the active site was done by picking the hollow having the utmost hydrophobic surface area of the receptor. The GA docking method was selected for the docking simulation. The virtual docking for all the conformers was conducted at elucidated cavity of the receptor. The fixed parameters of the docking simulation were the number of placements: 30 , rotation angles $30^{\circ}$ comprehensive method, scoring task: docking score. The rotation of the analogs to the divergent postures was obtained by the rotation angle. Besides docking simulation, the premier docked conformers of each ligand and receptor was transpired and assembled by elucidating the results of

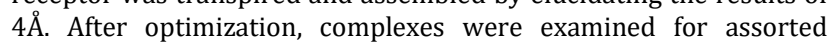


interSactions of the ligand with the receptor such as hydrogen bonding, hydrophobic bonding and van der Waal's interaction [18].

\section{In vitro anticancer evaluation}

The cell lines were grown on Roswell Park Memorial Institute 1640 (RPMI0-1640) media that contains fetal bovine serum (10\%) and $L$ glutamine $(2 \mathrm{mmol})$. Ninety-six well microtiter plates in $100 \mu \mathrm{l}$ at the solidity of plates were used to inoculate the cells depending upon the multiplication time of cell lines. After the cell inoculation process, microtiter plates were incubated for $24 \mathrm{~h}$, the conditions were sustained as $37{ }^{\circ} \mathrm{C}, 5 \% \quad \mathrm{CO}_{2}, 95 \%$ air and $100 \%$ Relative Humidity (Rh) before addition of synthesized compounds. The stock solution of the synthesized analogs was prepared at $100 \mathrm{mg} / \mathrm{kg}$ and was further diluted to $1 \mathrm{mg} / \mathrm{ml}$ with water and kept in chilled condition before use. Further, one fraction of chilled concentrate $(1 \mathrm{mg} / \mathrm{ml})$ was liquefied and was attenuated to $100,200,400$ and $800 \mu \mathrm{g} / \mathrm{ml}$ respectively with whole media comprising of standard drug. A portion of $10 \mu \mathrm{g}$ of dilutions containing diverse drugs was added to the suitable wells previously having $90 \mu \mathrm{l}$ media resulting in the ultimate drug concentrations that are $10,20,40$ and $80 \mu \mathrm{g} / \mathrm{ml}$ respectively.

After the addition of the compounds, plates were incubated in the standard conditions for $48 \mathrm{~h}$ and assay was discontinued after inclusion of trichloroacetic acid (TCA). An in-situ condition was maintained with mixing $50 \mu \mathrm{l}$ of chilled TCA gently (10\% of TCA) followed by further incubation for $1 \mathrm{~h}$ at the temperature of $4{ }^{\circ} \mathrm{C}$. Removal of resilient liquid was discarded; plates were washed with water 4-6 times and were dried under air. Sulforhodamine B (SRB) solution at the concentrations of $50 \mu \mathrm{l}$ at $0.4 \%(\mathrm{w} / \mathrm{v})$ in $1 \%$ acetic acid was appended to every well; $20 \mathrm{~min}$. the incubation period of these plates was obtained at room temperature. Once the dying process is completed the portion of unbound dye was recuperated and the unconsumed dye was omitted by washing 4-5 times with acetic acid in the concentration of $1 \%$ acetic acid. The plates were dried under the influence of air. Calculation of the percentage growth in plate sequence was compared to reference wells. Percent development of cells was revealed in terms of the proportion (ratio) of the mean absorbance of the well to the mean absorbance of the reference well X 100. By applying measurements of 6 absorbance [time 0], control growth (C), and the test in the presence of 4 concentration extents (Ti)], the \% growth was calculated at the concentration extent of every single drug [19-20].

$$
\left[\frac{T i}{C}\right] * 100 \%
$$

\section{In silico ADMET study}

For the identification and optimization of lead generation, in silico ADMET (absorption, distribution, metabolism, excretion, and toxicity) properties plays an essential role; ADMET SAR is open source software available online and was employed to predict the ADMET parameters. Here, the ADMET profile is estimated for the different analogs using the online server (www. lmmd. ecust. edu. cn, accessed on $5^{\text {th }}$ March 2017) [20-21].

\section{RESULTS AND DISCUSSION}

\section{Characterization study}

Fourteen triazine derivatives MA- 1 to MA-14 were synthesized. The yield of final compounds were obtained in the range of $50.00 \%$ to $90.04 \%$. Recrystallization was performed using ethanol. Structural characterization of synthesized analogues was done by IR, ${ }^{1} \mathrm{H}-\mathrm{NMR}$ and mass spectroscopy. The results of IR spectra confirm that all the compounds shows stretching under respective frequency within the range i. e; C-C Stretch (1500-1400 $\left.\mathrm{cm}^{-1}\right)$, C-N Stretch $(1250-1335 \mathrm{~cm}$ $\left.{ }^{1}\right), \mathrm{S}-\mathrm{C}=\mathrm{O}\left(1668-1705 \mathrm{~cm}^{-1}\right)$ etc. ${ }^{1} \mathrm{H}$ NMR spectra shows that protons of $S-5 \mathrm{H}-[1,2.4]$ triazino[5,6-b]indol-3 were observed in the aromatic region under the range of 6.8-7.2 $\delta \mathrm{ppm}$. Further, Mass spectra of compounds also confirms synthesized compounds.

\section{$S-5 H-[1,2,4]$-triazino ethanethioate (MA-1) \\ $(5,6-b)$ \\ indol-3-yl-3, \\ 4-biphenyl-}

Molecular formula; $\mathrm{C}_{24} \mathrm{H}_{20} \mathrm{~N}_{4} \mathrm{OS}$ (396.10), MP: $118-120^{\circ} \mathrm{C}$; yield 74.04\%; $\mathrm{R}_{\mathrm{f}}=0.69$ (toluene: EtOAc $\left.\{4: 1\}\right) ; \operatorname{IR} \operatorname{vmax}\left(\mathrm{cm}^{-1}\right)(\mathrm{KBr}): 1297$,
1665, 3069, 3305; ${ }^{1} \mathrm{H}-\mathrm{NMR}\left(\delta\right.$ ppm; $\left.\mathrm{CDCl}_{3}\right): 2.30(s, 3 \mathrm{H},-\mathrm{CH} 3), 4.27(s$, 2H,-CH2), 6.87-7.31 ( $m, 8 \mathrm{H}$, biphenyl), 7.31-7.69 ( $m, 3 \mathrm{H},-\mathrm{Ar})$.

\section{$S$-5H-[1,2,4]-triazino(5,6-b)indol-3-yl 2,4-dichlorobenzothioate (MA-2)}

Molecular formula: $\mathrm{C}_{16} \mathrm{H}_{8} \mathrm{Cl}_{2} \mathrm{~N}_{4} \mathrm{OS}$ (373.98); MP: $275^{\circ} \mathrm{C}$; yield: $80 \%$; $\mathrm{R}_{\mathrm{f}}: 0.77$ (toluene: EtOAc \{4:1\}); IR vmax $\left(\mathrm{cm}^{-1}\right)(\mathrm{KBr}): 1475.28$, 1308.46, $1700.91,599.62$; ${ }^{1} \mathrm{H}-\mathrm{NMR}$ ( $\delta \mathrm{ppm}$; DMSO): 7.38-7.85 (m, $3 \mathrm{H},-\mathrm{Ar})$, 7.00-7.80 ( $m, 4 \mathrm{H}$ phenyl), ESI-MS: $m / z 374.19(\mathrm{M}+\mathrm{H})^{+}$.

\section{$S$-5H-[1, 2, 4] triazino[5,6-b]indol-3-yl 3-bromobenzothioate (MA-3)}

Molecular formula: $\mathrm{C}_{16} \mathrm{H}_{9} \mathrm{BrN}_{4} \mathrm{OS}$ (385.24); MP: $276^{\circ} \mathrm{C}$; yield: $77 \%$; $\mathrm{R}_{\mathrm{f}}: 0.68$ (toluene: EtOAc \{4:1\}); IR vmax $\left(\mathrm{cm}^{-1}\right)(\mathrm{KBr}): 1479.81$, 1311.36, 1698.34, 617.10; ${ }^{1} \mathrm{H}-\mathrm{NMR}$ ( $\delta$ ppm; DMSO): 7.38-7.91 $(m, 4 \mathrm{H},-\mathrm{Ar}), 7.00-7.55\left(m, 4 \mathrm{H}\right.$ phenyl) ESI-MS: $m / z 384.8(\mathrm{M}-\mathrm{H})^{+}$.

$S$-5H-[1,2,4] triazino[5,6-b]indol-3-yl 4-chlorobenzothioate (MA-4)

Molecular formula: $\mathrm{C}_{16} \mathrm{H}_{9} \mathrm{ClN}_{4} \mathrm{OS}$ (340.79); MP: $277{ }^{\circ} \mathrm{C}$; yield: $88 \%$; $\mathrm{R}_{\mathrm{f}}$ : 0.55(toluene: EtOAc $\left.\{4: 1\}\right)$; IR $\operatorname{vmax}\left(\mathrm{cm}^{-1}\right)(\mathrm{KBr}): 1478.33$, 1312.06, 1698.34, 619.10; ${ }^{1} \mathrm{H}-\mathrm{NMR}$ ( $\delta \mathrm{ppm}$; DMSO): 7.36-8.00 ( $\mathrm{m}$, $4 \mathrm{H},-\mathrm{Ar}), 7.05-7.43$ ( $m, 5 H$ phenyl) ESI-MS: $m / z 340.02(\mathrm{M}+\mathrm{H})^{+}$.

\section{$\boldsymbol{S}$-5H-[1,2,4] triazino[5,6-b]indol-3-yl benzothioate (MA-5)}

Molecular formula: $\mathrm{C}_{17} \mathrm{H}_{12} \mathrm{~N}_{4} \mathrm{OS}(306.6)$; $\mathrm{MP}:>300^{\circ} \mathrm{C}$; yield: $77 \%$; $\mathrm{R}_{\mathrm{f}}=$ 0.55(toluene: EtOAc $\{4: 1\})$; IR vmax $\left(\mathrm{cm}^{-1}\right)(\mathrm{KBr}): 1475.28,1306.46$, 1700.91, 599.62; ${ }^{1} \mathrm{H}-\mathrm{NMR}$ ( $\delta$ ppm; DMSO): 7.0-7.55 ( $m, 4 \mathrm{H},-$ Indole), 7.5-7.97 $(m, 5 H,-\mathrm{Ar}) ; m / z=321.08(\mathrm{M}+\mathrm{H})^{+}$

\section{$S$-5H-[1,2,4] triazino [5,6-b] indol-3-yl 4-methylbenzothioate (MA-6)}

Molecular formula: $\mathrm{C}_{17} \mathrm{H}_{12} \mathrm{~N}_{4} \mathrm{OS}$ (320.37); MP: $>300{ }^{\circ} \mathrm{C}$; yield: $90 \%$; $\mathrm{R}_{\mathrm{f}}$ $=$ 0.58(toluene: EtOAc \{4:1\}); IR vmax $\left(\mathrm{cm}^{-1}\right)(\mathrm{KBr}):$ 1500.35, 1281.47, 1714.41, 2988.05; ${ }^{1} \mathrm{H}-\mathrm{NMR}$ ( $\delta$ ppm; DMSO): $2.35\left(s,-\mathrm{CH}_{3}\right)$, 7.33-7.75 (m,-Ar), 7.00-7.85 ( $m$, indole).

\section{$S-5 H-[1,2,4]$ triazino [5,6-b] indol-3-yl 3-methylbenzothioate (MA-7)}

Molecular formula: $\mathrm{C}_{17} \mathrm{H}_{12} \mathrm{~N}_{4} \mathrm{OS}$ (320.37); MP: $>300{ }^{\circ} \mathrm{C}$; yield: $70 \%$; $\mathrm{R}_{\mathrm{f}}$ : 0.62(toluene: EtOAc $\left.\{4: 1\}\right)$; IR $\operatorname{vmax}\left(\mathrm{cm}^{-1}\right)(\mathrm{KBr}):$ 1431.69, 1347.03, 1714.41, 2891.91, ${ }^{1} \mathrm{H}-\mathrm{NMR}$ ( $\delta \mathrm{ppm}$; DMSO): $2.51\left(\mathrm{~s},-\mathrm{CH}_{3}\right)$, 7.25-7.85 ( $m$,-Ar), 7.06-7.88 ( $m$, indole).

\section{$S$-5H-[1,2,4]triazino $[5,6-b]$ indol-3-yl 3,4,5-trihydroxybenzo- thioate (MA-8)}

Molecular formula: $\mathrm{C}_{16} \mathrm{H}_{10} \mathrm{~N}_{4} \mathrm{O}_{4} \mathrm{~S}$ (354.34); MP: $>300{ }^{\circ} \mathrm{C}$; yield: $70 \%$; $\mathrm{R}_{\mathrm{f}}$ : 0.56 (toluene: EtOAc \{4:1\}); IR vmax $\left(\mathrm{cm}^{-1}\right)(\mathrm{KBr}):$ 1474.31, 1310.39, 1696.91, 3115.44, ${ }^{1} \mathrm{H}-\mathrm{NMR}$ ( $\delta \mathrm{ppm}$; DMSO): $5.0(\mathrm{~m},-\mathrm{OH})$, 7.05-7.40 ( $m,-\mathrm{Ar}$ ), 7.0-7.85 ( $m$, indole).

\section{$S-5 H-[1,2,4]$ triazino[5,6-b]indol-3-yl 3-methoxybenzothioate} (MA-9)

Molecular formula: $\mathrm{C}_{16} \mathrm{H}_{10} \mathrm{~N}_{4} \mathrm{O}_{4} \mathrm{~S}$ (336.37); MP: $>300{ }^{\circ} \mathrm{C}$; yield: $70 \%$; $\mathrm{R}_{\mathrm{f}}$ : 0.56 (toluene: EtOAc \{4:1\}); IR vmax $\left(\mathrm{cm}^{-1}\right)(\mathrm{KBr}): 1477.21$, 1300.36, 1686.88, 3120.422, ${ }^{1} \mathrm{H}$-NMR ( $\delta$ ppm; DMSO): 5.0 ( $\left.m,-\mathrm{OH}\right)$, 7.05-7.40 ( $m,-\mathrm{Ar}$ ), 7.0-7.85 ( $m$, indole).

\section{$S$-5H-[1,2,4]triazino[5,6-b]indol-3-yl pyridine-3-carbothioate (MA-10)}

Molecular formula: $\mathrm{C}_{15} \mathrm{H}_{9} \mathrm{~N}_{5} \mathrm{OS}$ (307.04); MP: $>300{ }^{\circ} \mathrm{C}$; yield: $88 \%$; $\mathrm{R}_{f}$ : 0.68 (toluene: EtOAc \{4:1\}); IR vmax $\left(\mathrm{cm}^{-1}\right)(\mathrm{KBr}): 1475.28,1306.46$, 1700.91, ${ }^{1} \mathrm{H}-\mathrm{NMR}$ ( $\delta$ ppm; DMSO): 7.00-7.85 ( $m, 4 \mathrm{H}$, indole), 7.497.64 ( $m,-$ Ar).

\section{$S$-5H-[1,2,4]triazino[5,6-b]indol-3-yl 3-aminobenzothioate (MA- 11)}

Molecular formula: $\mathrm{C}_{16} \mathrm{H}_{11} \mathrm{~N}_{5} \mathrm{OS}$ (321.37); MP: $>300{ }^{\circ} \mathrm{C}$; yield: $90 \%$; $\mathrm{R}_{\mathrm{f}}$ : 0.68 (toluene: EtOAc \{4:1\}); IR vmax $\left(\mathrm{cm}^{-1}\right)(\mathrm{KBr}): 1499.05$, 1384.43, 1718.15, 1600.65; ${ }^{1} \mathrm{H}-\mathrm{NMR}$ ( $\delta$ ppm; DMSO): 7.06-7.89 ( $\mathrm{m}$, $4 \mathrm{H}$, indole), 7.43-7.68 ( $m$,-Ar) 4.32 (s, aromatic-NH).

$\boldsymbol{S}$-5H-[1,2,4] triazino[5,6-b]indol-3-yl 5-aminobenzothioate (MA-12)

Molecular formula: $\mathrm{C}_{16} \mathrm{H}_{11} \mathrm{~N}_{5} \mathrm{OS}$ (321.07); MP: $>300{ }^{\circ} \mathrm{C}$; yield: $93 \%$; Rf: 0.68 (toluene: EtOAc \{4:1\}); IR vmax $\left(\mathrm{cm}^{-1}\right)(\mathrm{KBr}):$ 1500.35, 
1381.28, 1714.41, 1599.66; ${ }^{1} \mathrm{H}-\mathrm{NMR}$ ( $\delta \mathrm{ppm}$; DMSO): 7.00-7.85( $\mathrm{m}$, $4 \mathrm{H}$, indole), 7.49-7.64 (m,-Ar) $4.0(\mathrm{~s}$, aromatic-NH).

\section{$S-5 H-[1,2,4]$ triazino[5,6-b]indol-3-yl 2-hydroxybenzthioate (MA-13)}

Molecular formula: $\mathrm{C}_{16} \mathrm{H}_{10} \mathrm{~N}_{4} \mathrm{O}_{2} \mathrm{~S}$ (322.35); MP: $>300{ }^{\circ} \mathrm{C}$; yield: $83 \%$; $\mathrm{R}_{\mathrm{f}:} 0.50$ (toluene: EtOAc $\left.\{4: 1\}\right)$; IR $\operatorname{vmax}\left(\mathrm{cm}^{-1}\right)(\mathrm{KBr}): 1459.85$, 1297.86, 1699.34, 3233.88; ${ }^{1} \mathrm{H}-\mathrm{NMR}$ ( $\delta$ ppm; DMSO): 7.00-7.85( $\mathrm{m}$, $4 \mathrm{H}$, indole), 6.92-7.48 (m,-Ar), $5.0(\mathrm{~s}, \mathrm{Ar}-\mathrm{OH})$.

\section{$S$-5H-[1,2.4] triazino[5,6-b]indol-3-yl 3-nitrobenzthioate (MA-14)}

Molecular formula: $\mathrm{C}_{16} \mathrm{H}_{10} \mathrm{~N}_{4} \mathrm{O}_{2} \mathrm{~S}$ (322.35); MP: $>300{ }^{\circ} \mathrm{C}$; yield: $83 \%$; $\mathrm{R}_{\mathrm{f}}: 0.50$ (toluene: EtOAc $\left.\{4: 1\}\right) ; \mathrm{IR} \operatorname{vmax}\left(\mathrm{cm}^{-1}\right)(\mathrm{KBr}): 1259.29$,
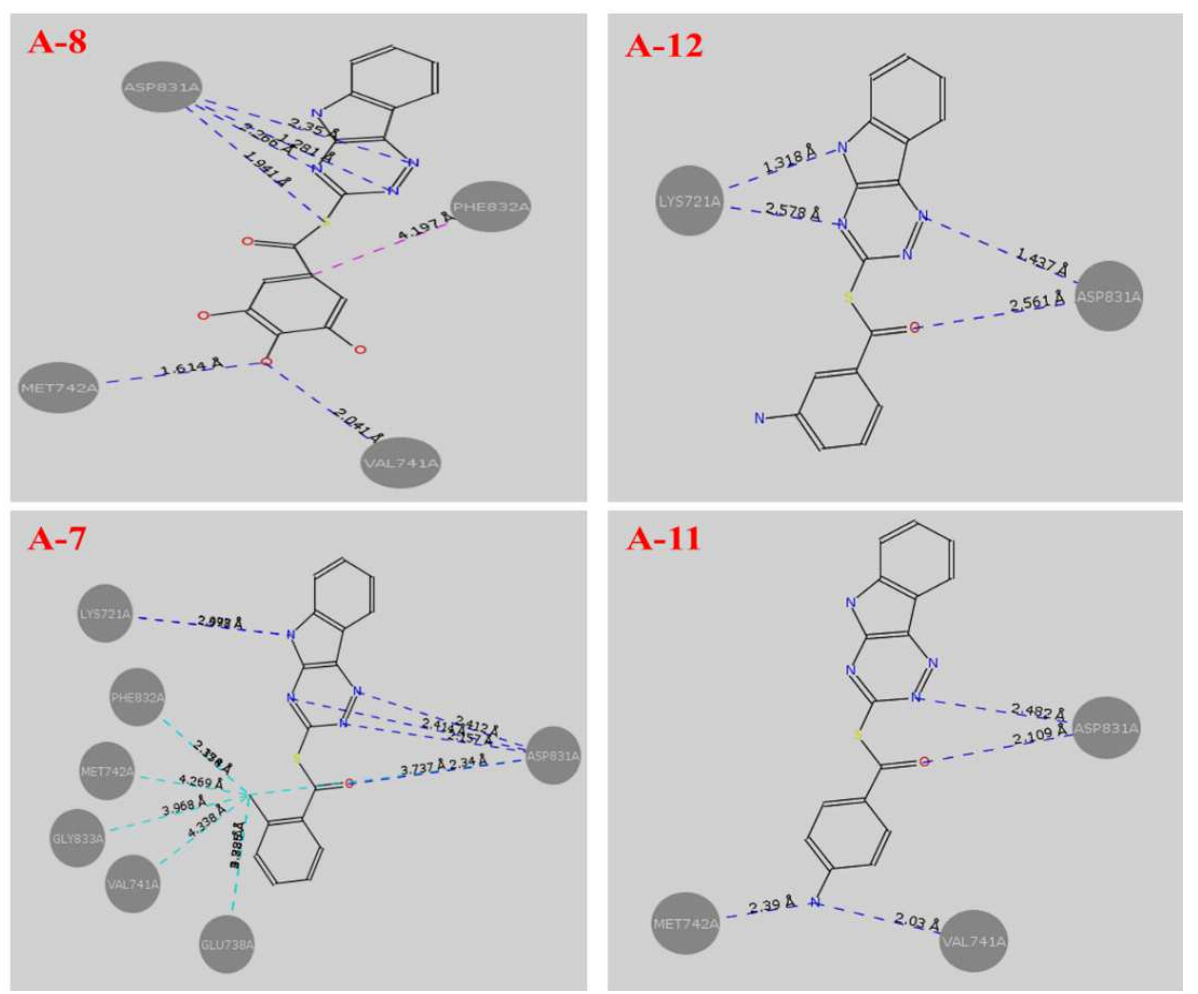
6.92-7.48 (m,-Ar).

\section{Molecular docking} shown in the fig. 1.

1468.53, 1699.94; ${ }^{1} \mathrm{H}-\mathrm{NMR}$ ( $\delta$ ppm; DMSO): 7.00-7.85( $m, 4 \mathrm{H}$, indole),

To investigate the interaction between epidermal growth factor receptor (EGFR) and synthesized derivatives; molecular docking study was carried out. Active compound MA-8 binds with ASP813A and VAL741A; MA-12 binds with ASP8134A, HIS811A and PHE832A; MA-13 binds with ASP8134A and PHE832A; MA-14 binds with ASP813A and LYS721A amino acids. Interaction of compounds with the crystal structure of epidermal growth factor receptor (EGFR) is

Fig. 1: Docking interaction of compounds with 1M17

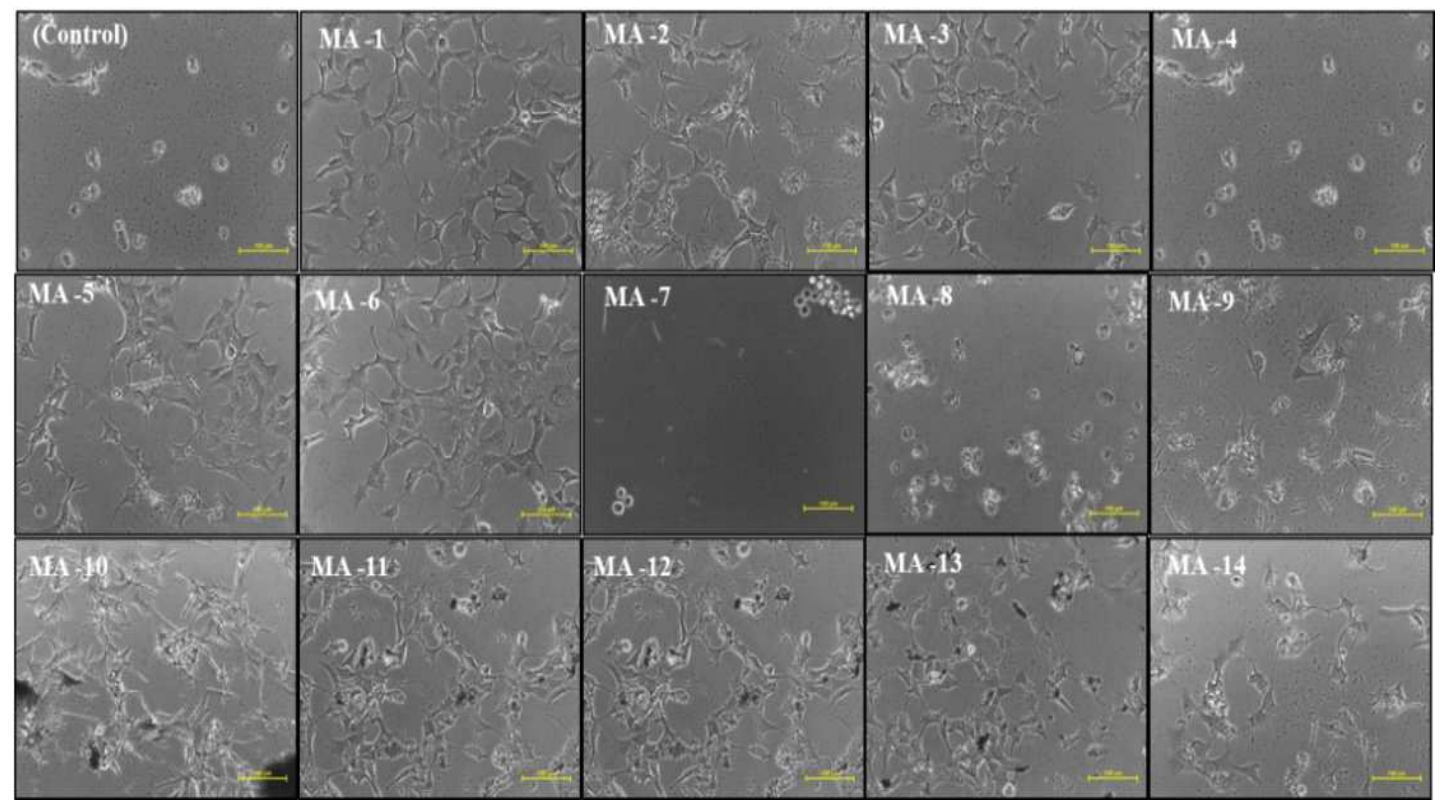

Fig. 2: Phase contrast images of MCF-7 cells showing morphological changes following the treatment with synthesized compounds 


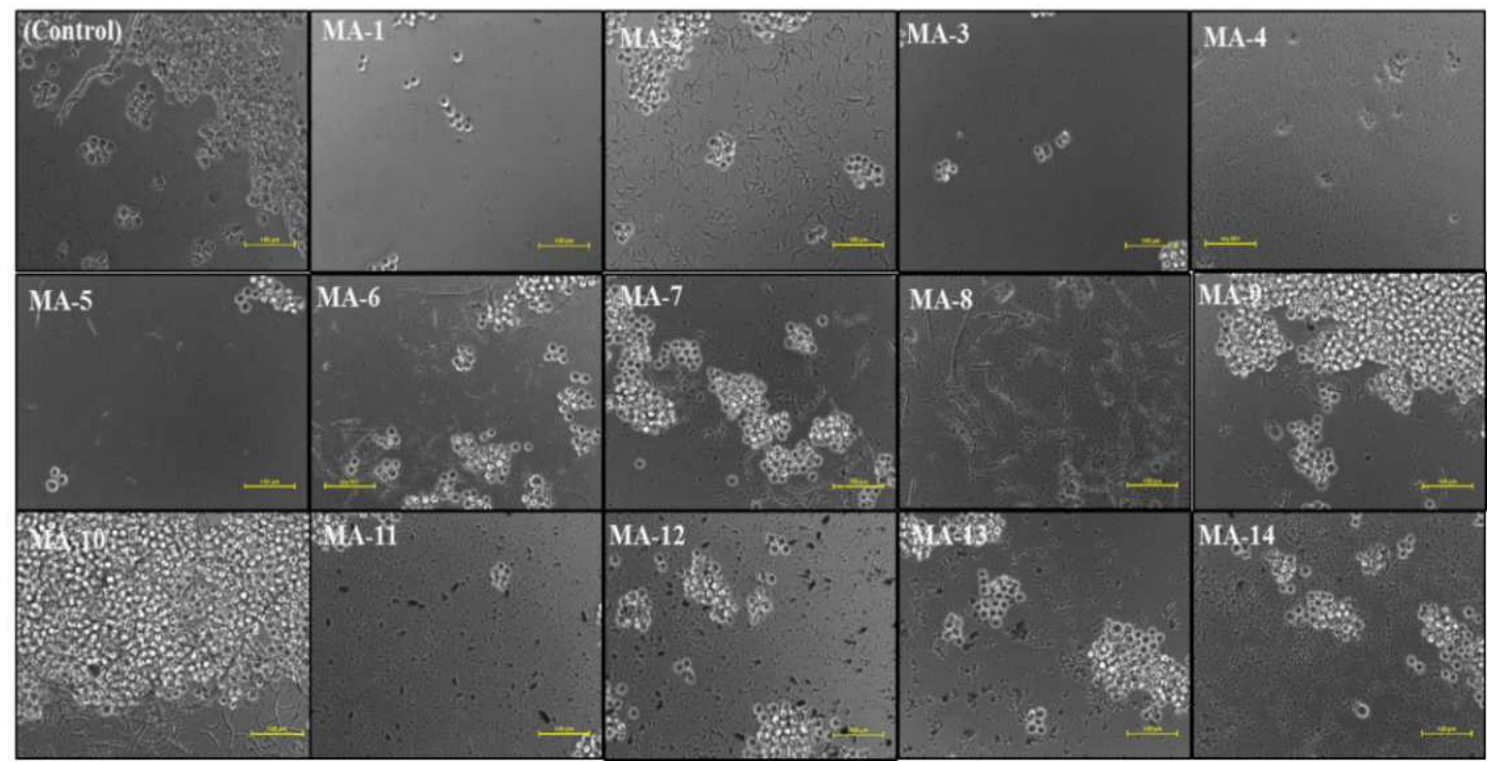

Fig. 3: Phase contrast images of K-562 cells showing morphological changes following the treatment with synthesized compounds

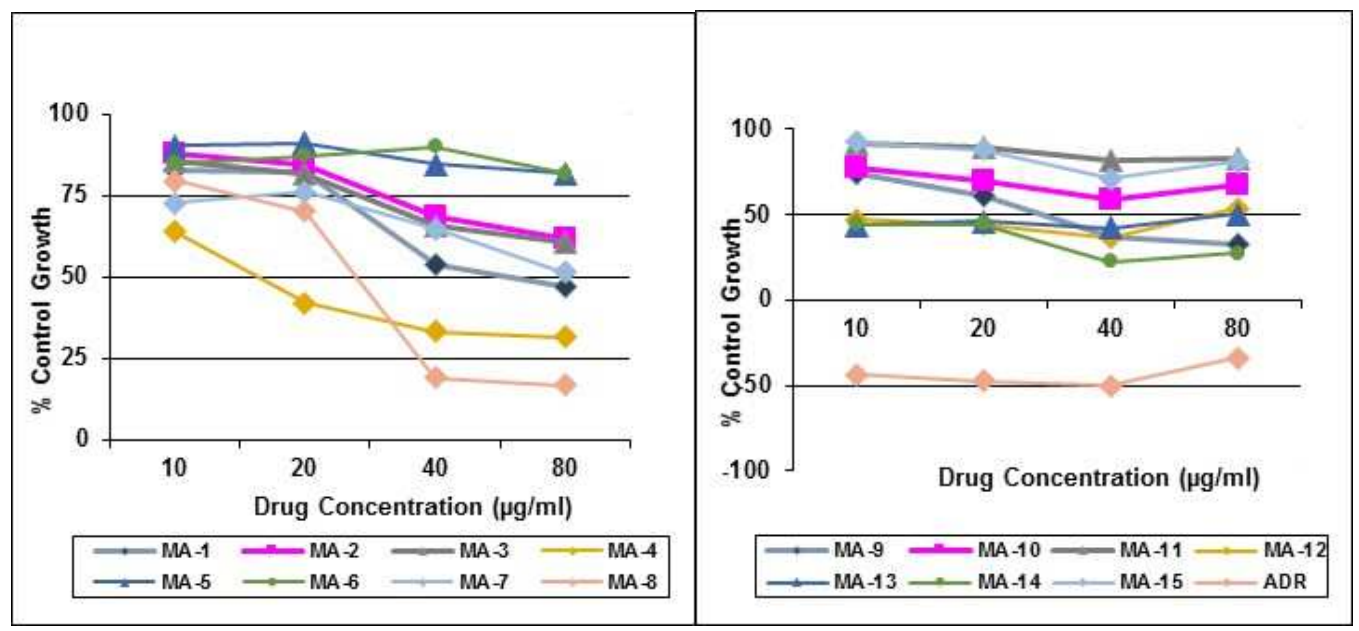

Fig. 4: Growth Curve: Human Breast Cancer Cell Line (MCF-7)

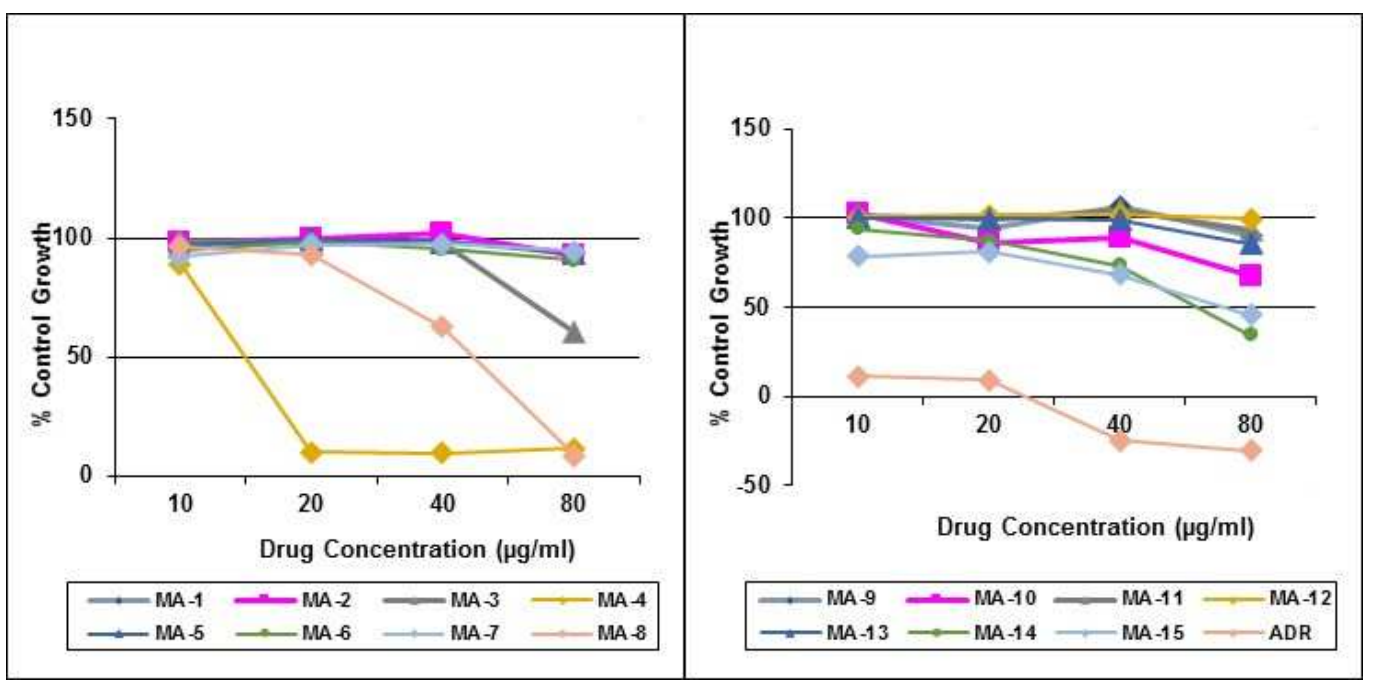

Fig. 5: Growth Curve: Human Leukaemia Cell Line (K-562) 
Table 1: Cytotoxicity on human breast cancer cell line MCF-7

\begin{tabular}{|c|c|c|c|c|c|c|c|c|c|c|c|c|c|c|c|c|}
\hline \multicolumn{17}{|c|}{ Human breast cancer cell line MCF-7 } \\
\hline \multicolumn{17}{|c|}{ \% Control growth } \\
\hline \multicolumn{17}{|c|}{ Drug concentrations $(\mu \mathrm{g} / \mathrm{ml})$} \\
\hline Compound & Expe & ment N & & & Exper & nent $\mathbf{N}$ & & & Exper & ient No. & & & Avera & e value & & \\
\hline & 10 & 20 & 40 & 80 & 10 & 20 & 40 & 80 & 10 & 20 & 40 & 80 & 10 & 20 & 40 & 80 \\
\hline MA-1 & 80.4 & 79.0 & 64.1 & 58.4 & 75.3 & 76.4 & 69.5 & 53.0 & 91.6 & 91.0 & 27.8 & 30.1 & 82.5 & 82.1 & 53.8 & 47.2 \\
\hline MA-2 & 87.7 & 86.8 & 75.0 & 65.4 & 82.8 & 83.5 & 72.4 & 62.2 & 93.1 & 82.6 & 58.8 & 57.3 & 87.9 & 84.3 & 68.7 & 61.7 \\
\hline MA-3 & 80.5 & 73.3 & 56.3 & 40.2 & 80.8 & 75.4 & 55.4 & 42.4 & 95.2 & 95.6 & 84.6 & 98.7 & 85.5 & 81.4 & 65.4 & 60.4 \\
\hline MA-4 & 45.9 & 6.9 & 1.4 & 7.6 & 39.7 & 7.7 & 2.9 & 11.2 & 105.5 & 110.9 & 94.8 & 75.9 & 63.7 & 41.8 & 33.0 & 31.5 \\
\hline MA-5 & 75.8 & 79.7 & 74.0 & 72.7 & 78.2 & 79.1 & 70.6 & 68.7 & 116.3 & 114.2 & 109.2 & 103.5 & 90.1 & 91.0 & 84.6 & 81.6 \\
\hline MA-6 & 76.6 & 77.3 & 81.0 & 79.8 & 76.7 & 80.8 & 81.3 & 78.5 & 101.5 & 103.2 & 107.4 & 87.1 & 84.9 & 87.1 & 89.9 & 81.8 \\
\hline MA-7 & 86.9 & 81.8 & 71.2 & 58.3 & 77.7 & 79.2 & 73.2 & 60.4 & 53.1 & 67.3 & 49.7 & 34.9 & 72.6 & 76.1 & 64.7 & 51.2 \\
\hline MA-8 & 78.2 & 68.4 & 8.8 & 11.8 & 80.8 & 76.9 & 14.2 & 11.8 & 79.2 & 64.9 & 33.8 & 26.7 & 79.4 & 70.1 & 18.9 & 16.7 \\
\hline MA-9 & 69.7 & 56.4 & 37.8 & 34.6 & 72.7 & 61.9 & 41.8 & 39.9 & 79.0 & 62.7 & 31.6 & 23.4 & 73.8 & 60.3 & 37.1 & 32.7 \\
\hline MA-10 & 77.0 & 71.0 & 67.3 & 86.0 & 79.7 & 74.2 & 73.8 & 89.4 & 75.5 & 62.6 & 35.4 & 28.2 & 77.4 & 69.3 & 58.8 & 67.9 \\
\hline MA-11 & 87.2 & 81.4 & 74.5 & 65.6 & 88.0 & 84.9 & 77.9 & 72.0 & 98.8 & 102.8 & 93.1 & 109.7 & 91.3 & 89.7 & 81.8 & 82.4 \\
\hline MA-12 & 92.4 & 91.2 & 78.5 & 83.2 & 95.0 & 89.1 & 80.0 & 92.2 & -46.3 & -48.3 & -49.3 & -15.2 & 47.0 & 44.0 & 36.4 & 53.4 \\
\hline MA-13 & 85.3 & 93.9 & 91.8 & 72.9 & 92.2 & 90.8 & 88.0 & 88.5 & -46.3 & -46.5 & -53.2 & -10.5 & 43.8 & 46.1 & 42.2 & 50.3 \\
\hline MA-14 & 89.3 & 75.5 & 53.4 & 42.0 & 89.6 & 86.1 & 69.2 & 57.7 & -46.5 & -29.1 & -56.0 & -18.4 & 44.1 & 44.2 & 22.2 & 27.1 \\
\hline Adriamycin & - & -47.3 & -50.2 & -33.7 & -43.4 & -47.3 & -50.2 & -33.7 & -43.4 & -47.3 & -50.2 & -33.7 & -43.4 & -47.3 & -50.2 & -33.7 \\
\hline
\end{tabular}

Table 2: Cytotoxicity on human breast cancer cell line K-562

\begin{tabular}{|c|c|c|c|c|c|c|c|c|c|c|c|c|c|c|c|c|}
\hline \multicolumn{17}{|c|}{ Human breast cancer cell line K-562 } \\
\hline \multicolumn{17}{|c|}{ \% Control growth } \\
\hline \multicolumn{17}{|c|}{ Drug concentrations $(\mu \mathrm{g} / \mathrm{ml})$} \\
\hline Compound & Expe & ment & D. 1 & & Experi & nent No. & & & Exper & nent No & & & Avera & values & & \\
\hline & 10 & 20 & 40 & 80 & 10 & 20 & 40 & 80 & 10 & 20 & 40 & 80 & 10 & 20 & 40 & 80 \\
\hline MA-1 & 91.8 & 96.3 & 96.1 & 91.7 & 100.9 & 100.4 & 99.2 & 99.0 & 98.3 & 98.7 & 101.9 & 89.5 & 97.0 & 98.4 & 99.0 & 93.4 \\
\hline MA-2 & 98.6 & 98.3 & 102.0 & 89.5 & 94.7 & 101.6 & 100.7 & 97.9 & 101.9 & 99.7 & 104.0 & 89.9 & 98.4 & 99.9 & 102.2 & 92.5 \\
\hline MA-3 & 98.2 & 97.3 & 95.0 & 47.6 & 101.6 & 99.2 & 96.9 & 67.8 & 96.9 & 98.0 & 101.8 & 65.5 & 98.9 & 98.2 & 97.9 & 60.3 \\
\hline MA-4 & 83.8 & 8.1 & 9.8 & 13.5 & 91.5 & 7.1 & 0.4 & 9.2 & 92.4 & 15.0 & 18.4 & 12.0 & 89.2 & 10.0 & 9.5 & 11.6 \\
\hline MA-5 & 91.7 & 96.5 & 95.8 & 85.0 & 100.0 & 100.5 & 95.4 & 99.6 & 98.4 & 100.9 & 103.2 & 96.7 & 96.7 & 99.3 & 98.1 & 93.8 \\
\hline MA-6 & 90.9 & 96.6 & 91.7 & 72.2 & 96.1 & 98.0 & 93.7 & 99.9 & 98.0 & 101.8 & 100.0 & 99.5 & 95.0 & 98.8 & 95.1 & 90.5 \\
\hline MA-7 & 92.6 & 94.2 & 92.5 & 87.6 & 96.4 & 98.2 & 96.3 & 98.2 & 88.0 & 99.1 & 104.2 & 97.5 & 92.3 & 97.2 & 97.7 & 94.4 \\
\hline MA-8 & 84.7 & 86.8 & 67.0 & 22.6 & 101.9 & 95.5 & 61.8 & 13.0 & 103.1 & 96.2 & 59.1 & -10.2 & 96.6 & 92.9 & 62.6 & 8.5 \\
\hline MA-9 & 96.5 & 82.7 & 105.7 & 95.9 & 102.7 & 98.6 & 102.1 & 89.8 & 103.9 & 101.6 & 111.1 & 83.9 & 101.0 & 94.3 & 106.3 & 89.9 \\
\hline MA-10 & 96.7 & 73.4 & 96.8 & 84.8 & 102.1 & 96.6 & 82.1 & 84.2 & 108.6 & 88.5 & 87.5 & 32.0 & 102.4 & 86.2 & 88.8 & 67.0 \\
\hline MA-11 & 94.5 & 99.3 & 105.5 & 92.8 & 103.5 & 97.3 & 99.6 & 95.4 & 106.9 & 101.4 & 107.0 & 90.3 & 101.6 & 99.3 & 104.0 & 92.8 \\
\hline MA-12 & 98.4 & 96.7 & 101.2 & 97.7 & 100.0 & 101.2 & 99.5 & 99.1 & 103.8 & 106.4 & 103.9 & 100.9 & 100.7 & 101.4 & 101.5 & 99.2 \\
\hline MA-13 & 97.2 & 93.1 & 99.7 & 83.3 & 99.1 & 101.9 & 98.7 & 92.5 & 103.8 & 103.0 & 98.8 & 81.5 & 100.0 & 99.3 & 99.1 & 85.8 \\
\hline MA-14 & 90.5 & 82.8 & 69.0 & 28.3 & 97.1 & 96.5 & 82.4 & 47.1 & 94.4 & 82.5 & 67.9 & 27.1 & 94.0 & 87.3 & 73.1 & 34.1 \\
\hline Adriamycin & 12.4 & 7.5 & -20.5 & $\begin{array}{l}- \\
30.8\end{array}$ & 7.5 & 6.4 & -29.0 & $\begin{array}{l}- \\
26.0\end{array}$ & 13.6 & 13.5 & -24.2 & -34.0 & 11.2 & 9.1 & -24.6 & $\begin{array}{l}- \\
30.3\end{array}$ \\
\hline
\end{tabular}

Table 3: GI $\mathrm{I}_{50}$ values $(\mu \mathrm{g} / \mathrm{ml})$ of standard and test compounds

\begin{tabular}{|c|c|c|c|}
\hline \multirow[t]{2}{*}{ S. No. } & \multirow[t]{2}{*}{ Compound code } & \multicolumn{2}{|c|}{ Growth inhibition $50 \%\left(\mathrm{GI}_{50}\right)$ values $(\mu \mathrm{g} / \mathrm{ml})$} \\
\hline & & MCF-7 & K-562 \\
\hline 1. & MA-1 & 67.6 & $>80$ \\
\hline 2. & MA-2 & NE & $>80$ \\
\hline 3. & MA-3 & NE & $>80$ \\
\hline 4. & MA-4 & 17.2 & 10.4 \\
\hline 5. & MA-5 & $\mathrm{NE}$ & $>80$ \\
\hline 6. & MA-6 & NE & $>80$ \\
\hline 7. & MA-7 & 84.4 & NE \\
\hline 8. & MA-8 & 33.5 & 49.1 \\
\hline 9. & MA-9 & 39.3 & $>80$ \\
\hline 10. & MA-10 & $>80$ & $>80$ \\
\hline 11. & MA-11 & $>80$ & $>80$ \\
\hline 12. & MA-12 & $<10$ & $>80$ \\
\hline 13. & MA-13 & $<10$ & 63.2 \\
\hline 14. & MA-14 & $<10$ & 72.3 \\
\hline STD & Adriamycin & $<10$ & $<10$ \\
\hline
\end{tabular}

\section{In vitro anticancer evaluation}

The newly synthesized conjugates bearing various substituents on the triazine ring were screened for their cytotoxicity on two cell lines namely breast cancer (MCF-7) and leukaemia (K-562) cell lines using SRB assay; Adriamycin was used as the positive control. The results of cytotoxic activity against cell lines are represented in table 1 and table 2 . GI I $_{50}$ values for standard and test compounds are depicted in the table 3. Compounds MA-12 $\left(\mathrm{GI}_{50}\right.$ $=<10 \mu \mathrm{g} / \mathrm{ml}), \mathrm{MA}-13\left(\mathrm{GI}_{50}=<10 \mu \mathrm{g} / \mathrm{ml}\right)$ and $\mathrm{MA}-14\left(\mathrm{GI}_{50}=<10\right.$ $\mu \mathrm{g} / \mathrm{ml}$ ) were found to be the most active compounds against the breast cancer cell line and compound MA-4 (GI $\left.\mathrm{G}_{50}=10.4 \mu \mathrm{g} / \mathrm{ml}\right)$ showed the mild anticancer activity against the Adriamycin (positive control) as shown in fig. 2 and fig. 3. 
Table 4: Predicted ADMET-SAR properties of synthesized compounds

\begin{tabular}{|c|c|c|c|c|c|c|c|c|c|c|c|c|c|c|c|c|}
\hline 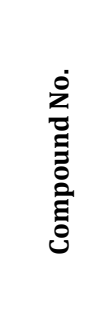 & 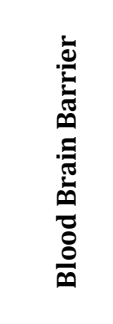 & 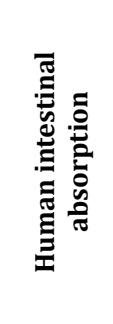 & 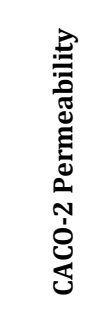 & 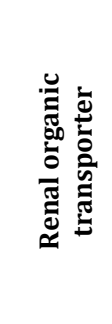 & 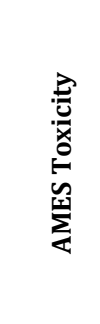 & 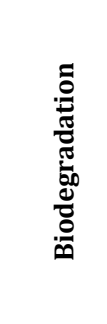 & 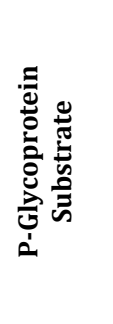 & 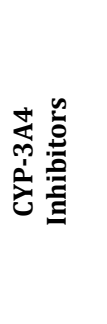 & 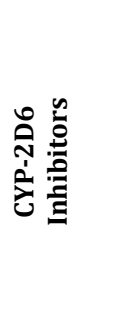 & 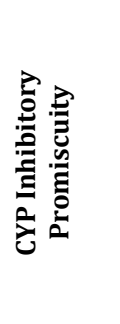 & 总 & 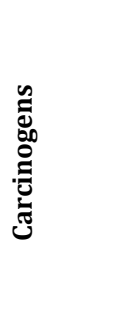 & 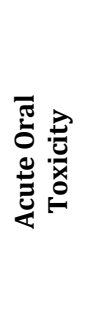 & 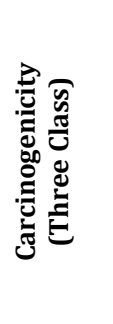 & 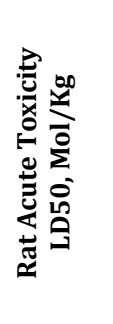 & 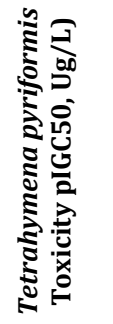 \\
\hline \multicolumn{17}{|c|}{ Probability } \\
\hline MA-1 & 0.9744 & 1.0000 & 0.5511 & 0.7590 & 0.5571 & 0.9967 & 0.7701 & 0.6981 & 0.8551 & 0.7045 & 0.9135 & 0.8556 & 0.6213 & 0.5884 & 2.3435 & 0.8682 \\
\hline MA-2 & 0.9493 & 1.0000 & 0.5171 & 0.7313 & 0.5784 & 1.0000 & 0.7670 & 0.6377 & 0.8950 & 0.6488 & 0.9018 & 0.8979 & 0.5761 & 0.6252 & 2.4510 & 1.1856 \\
\hline MA-3 & 0.9638 & 1.0000 & 0.5311 & 0.7328 & 0.5258 & 1.0000 & 0.7616 & 0.6899 & 0.6342 & 0.6601 & 0.9331 & 0.9105 & 0.6050 & 0.5773 & 2.4631 & 1.0988 \\
\hline MA-4 & 0.9646 & 1.0000 & 0.5130 & 0.7314 & 0.5455 & 1.0000 & 0.7088 & 0.6572 & 0.9826 & 0.5904 & 0.9003 & 0.8952 & 0.6185 & 0.6042 & 2.4294 & 1.0799 \\
\hline MA-5 & 0.9738 & 1.0000 & 0.5444 & 0.7492 & 0.5577 & 0.9882 & 0.7597 & 0.6516 & 0.8474 & 0.5498 & 0.9266 & 0.9223 & 0.6320 & 0.5720 & 2.3361 & 0.8416 \\
\hline MA-6 & 0.9676 & 1.0000 & 0.5509 & 0.7640 & 0.5474 & 0.9927 & 0.7597 & 0.7382 & 0.8982 & 0.5498 & 0.9266 & 0.9173 & 0.6778 & 0.5089 & 2.2955 & 0.7848 \\
\hline MA-7 & 0.9566 & 1.0000 & 0.5000 & 0.7659 & 0.5313 & 0.9927 & 0.6947 & 0.6941 & 0.9332 & 0.5292 & 0.9169 & 0.9214 & 0.6211 & 0.5279 & 2.4114 & 0.7633 \\
\hline MA-8 & 0.6011 & 0.9837 & 0.6440 & 0.8815 & 0.5577 & 0.9792 & 0.6908 & 0.6325 & 0.9118 & 0.5814 & 0.9178 & 0.8879 & 0.5480 & 0.5859 & 2.3483 & 0.7288 \\
\hline MA-9 & 0.9559 & 1.0000 & 0.5000 & 0.6978 & 0.5437 & 0.9605 & 0.5704 & 0.6316 & 0.7687 & 0.6950 & 0.9696 & 0.9343 & 0.5187 & 0.4758 & 2.3532 & 0.7727 \\
\hline MA-10 & 0.9738 & 1.0000 & 0.5444 & 0.7492 & 0.5577 & 0.9882 & 0.6670 & 0.6887 & 0.8622 & 0.5875 & 0.8585 & 0.9223 & 0.6320 & 0.5720 & 2.3361 & 0.8416 \\
\hline MA-11 & 0.9621 & 0.9954 & 0.5137 & 0.7974 & 0.6170 & 1.0000 & 0.7597 & 0.7382 & 0.8982 & 0.5498 & 0.9266 & 0.8684 & 0.6683 & 0.4508 & 2.3803 & 0.6039 \\
\hline MA-12 & 0.9621 & 0.9954 & 0.5137 & 0.7974 & 0.6170 & 1.0000 & 0.9597 & 0.6155 & 0.9135 & 0.7022 & 0.9403 & 0.8684 & 0.6683 & 0.4508 & 2.3803 & 0.6039 \\
\hline MA-13 & 0.9134 & 1.000 & 0.5641 & 0.7801 & 0.5360 & 0.9806 & 0.9597 & 0.6155 & 0.9135 & 0.7022 & 0.9403 & 0.8906 & 0.5402 & 0.5525 & 2.4074 & 0.9266 \\
\hline MA-14 & 0.9141 & 0.9875 & 0.5385 & 0.8366 & 0.8212 & 0.9843 & 0.6884 & 0.6462 & 0.6942 & 0.7118 & 0.9098 & 0.8074 & 0.5853 & 0.4511 & 0.6728 & 0.8016 \\
\hline
\end{tabular}


Further, only active compounds show significant interaction at the active site of receptor via hydrogen bonding to some important amino acids such as ASP, VAL, MET, LYS etc. Combination of different interactions leads to significant penetration of compounds at the active site of the receptor with good docking score. Hence, it is observed that molecular docking and anticancer activity are correlated with each other. The results of anticancer activity are depicted in fig. 2 and fig. 3 respectively.

\section{In silico ADMET SAR study}

Pharmacokinetic and pharmacodynamic profiling of the drug is one of the most important and critical Parameters; beginning with the site of administration, absorption into the systemic circulation, gesticulation in blood, elimination through urine. ADMET profiling of the synthesized triazine derivatives has been calculated with the help of online software database namely admet SAR. There are diverse parameters for determining the permeability of the drugs through various body barriers such as blood-brain barrier (BBB), human intestinal absorption, Caco- 2 cell permeation, absorption in the intestine, renal organic cation transport and AMES toxicity test. P-glycoprotein is the membrane efflux transporter which plays a critical role in the determination of pharmacokinetic [ADMEandT] profile of some therapeutic agents and in the multidrug resistance (MDR) phenomena. Recognition of the P-glycoprotein substrates is an important process in the early stage of the drug discovery. Cytochrome-P enzyme family (CYPs) is the essential enzyme which plays a critical role in detoxification of drugs and the biosynthesis of endogenous molecules. The direct or indirect correlation of the carcinogenicity of therapeutic agents with the properties of the molecule is an important feature in the pharmacokinetic profiling of the drugs. The results of carcinogenicity, mutagenicity from the admetSAR software have been listed in table 4. The triazine derivatives might be able to pass through the human intestinal barrier and could be absorbed from the intestine. Out of all the synthesized triazine derivatives, majority of the compounds do not show any kind of toxicity or mutagenicity.

\section{CONCLUSION}

A series of novel triazine derivatives were synthesized and chemical structure of all analogs was confirmed by IR, ${ }^{1} \mathrm{H}-\mathrm{NMR}$ and mass spectroscopy. The compounds were evaluated for anticancer activity using MCF-7 and K-562 cell lines. The compound MA-7, MA-8, MA12, MA-13 and MA-14 were found most active from the series against different cell lines. Further, synthesized compounds were docked on the crystal structure of EGFR receptor to identify the binding mode of interaction. Docking interaction and score supports triazine derivative as potent anticancer agents. Moreover, all compounds were subjected to in silico ADMET prediction using admetSAR online tool which suggest that all molecules from the series have appropriate ADMET properties to become a potential drug candidate. The results of a computational study and in vitro study strongly reveals that the synthesized compounds may be used for lead optimization for development of novel anticancer agents with a high degree of selectivity and specificity towards cancer cells.

\section{ACKNOWLEDGMENT}

We Thank 'Advanced Centre for Treatment, Research and Education in Cancer (ACTREC), Mumbai' for assistance with the cell line studies, Centre of Food Testing, Bharati Vidyapeeth (Deemed to Be University), Pune for providing LC-MS Services.

\section{CONTRIBUTIONS}

All the authors have contributed equally.

\section{CONFLICT OF INTERESTS}

There is no conflict of interest pertaining to this manuscript

\section{REFERENCES}

1. Subrahmanyam V, Vanita P, Jhansi K. A short note on cancer. J Carcinog Mutagen 2011;2:128.
2. McBee Jr WC, Gardiner AS, Edwards RP, Lesnock JL, Bhargava R. MicroRNA analysis in human papillomavirus (HPV)associated cervical neoplasia and cancer.J Carcinog Mutagen 2000;1. ).

3. Augustin HG. Antiangiogenic tumour therapy: will it work? Trends Pharm Sci 1998;19:216-22.

4. Hinck L, Näthke I. Changes in cell and tissue organization in cancer of the breast and colon. Curr Opi Cell Bio 2000;26:87-95.

5. Grivennikov SI, Greten FR, Karin M. Immunity, inflammation, and cancer. Cell 2010;140:883-99.

6. Racil Z, Razga F, Drapalova J, Buresova L, Zackova D, Palackova $\mathrm{M}$, et al. Mechanism of impaired glucose metabolism during nilotinib therapy in patients with chronic myelogenous leukemia. Haematol Haematol 2013;98:e124-6.

7. Sharma GN, Dave R, Sanadya J, Sharma P, Sharma KK. Various types and management of breast cancer: an overview. J Adv Pharm Tech Res 2010;1:109.

8. Perou, Charles M, Therese Sørlie, Michael B Eisen, Matt Van De Rijn, Stefanie S Jeffrey, Christian A Rees, et al. Molecular portraits of human breast tumours. Nature 2000;406:747.

9. Hallek M, Cheson BD, Catovsky D, Caligaris Cappio F, Dighiero G, Dohner H, et al. Guidelines for the diagnosis and treatment of chronic lymphocytic leukemia: a report from the international workshop on chronic lymphocytic leukemia updating the national cancer institute-working group 1996 guidelines. Blood 2008;111:5446-56.

10. Else M, Ruchlemer R, Osuji N, Del Giudice I, Matutes E, Woodman A, et al. Long remissions in hairy cell leukemia with purine analogs: a report of 219 patients with a median follow-up of $12.5 \mathrm{y}$.Cancer Interdisciplinary Int J Am Soc 2005;104:2442-8.

11. Wood ER, Truesdale AT, McDonald OB, Yuan D, Hassell A, Dickerson SH, et al. A unique structure for epidermal growth factor receptor bound to GW572016 (Lapatinib): relationships among protein conformation, inhibitor off-rate, and receptor activity in tumor cells. Can Res 2004;64:6652-9.

12. Campbell P, Morton PE, Takeichi T, Salam A, Roberts N, Proudfoot LE, et al. Epithelial inflammation resulting from an inherited loss-of-function mutation in EGFR. J Inves Derm 2014;134:2570-8.

13. Yun UJ, Sung JY, Park SY, Ye SK, Shim J, Lee JS, et al. Oncogenic role of rab escort protein 1 through EGFR and STAT3 pathway. Cell Death Dis 2017;8:e2621.

14. Zwick E, Hackel PO, Prenzel N, Ullrich A. The EGF receptor as central transducer of heterologous signalling systems. Trends Pharm Sci 1999;20:408-12.

15. Aswar UM, Kalshetti PP, Shelke SM, Bhosale SH, Bodhankar SL. Effect of newly synthesized 1, 2, 4-triazino [5, 6-b] indole-3thione derivatives on olfactory bulbectomy induced depression in rats. Asi Pac J Trop Biomed 2012;2:992-8.

16. Babu TMC, Rajesh SS, Bhaskar BV, Devi S, Rammohan A, Sivaraman T, et al. Molecular docking, molecular dynamics simulation, biological evaluation and 2D QSAR analysis of flavonoids from syzygium alternifolium as potent antihelicobacter pylori agents. RSC Adv 2017;7:18277-92.

17. Noolvi MN, Patel HM. A comparative QSAR analysis and molecular docking studies of quinazoline derivatives as tyrosine kinase (EGFR) inhibitors: A rational approach to anticancer drug design. J Saudi Chem Soc 2013;17:361-79.

18. Vichai V, Kirtikara K. Sulforhodamine B colorimetric assay for cytotoxicity screening. Nat Protoc 2006;1:1112.

19. Skehan P, Storeng R, Scudiero D, Monks A, McMahon J, Vistica $D$, et al. New colorimetric cytotoxicity assay for anticancer-drug screening. JNCI: J Nat Can Ins 1990;82:1107-12.

20. Variya B, Modi S, Savjani J, Patel S. In silico molecular docking and pharmacokinetic prediction of gallic acid derivatives as PPAR- $\gamma$ agonists. Int J Pharm Pharml Sci 2017;9:102-7.

21. Shinde MG, Modi SJ, Kulkarni VM. Synthesis, pharmacological evaluation, molecular docking and in silico ADMET prediction of nitric oxide releasing biphenyls as anti-inflammatory agents. J Appl Pharm Sci 2017; 7:37-47. 